\title{
FACE ANALYSIS AND SYNTHESIS FOR INTERACTIVE ENTERTAINMENT
}

\author{
Shoichiro IWASAWA ${ }^{* 1}$, Tatsuo YOTSUKURA ${ }^{* 2}$, Shigeo MORISHIMA ${ }^{* 2}$ \\ ${ }^{*}$ Telecommunication Advancement Organization ${ }^{* 2}$ Faculty of Engineering, Seikei University
}

\begin{abstract}
A stand-in is a common technique for movies and TV programs in foreign languages. The current stand-in that only substitutes the voice channel results awkward matching to the mouth motion. Videophone with automatic voice translation are expected to be widely used in the near future, which may face the same problem without lip-synchronized speaking face image translation. In this paper, we propose a method to track motion of the face from the video image and then replace the face part or only mouth part with synthesized one which is synchronized with synthetic voice or spoken voice. This is one of the key technologies not only for speaking image translation and communication system, but also for an interactive entertainment system. Finally, an interactive movie system is introduced as an application of entertainment system.
\end{abstract}

Key words: Face Image Synthesis, Lip Synchronization, Face Tracking, Interactive Movie

\section{INTRODUCTION}

Recently, CG technology is used in various media, such as TV programs, movies and amusements. The CG quality rapidly progresses in these several years, and has reached even at the same level as a photograph taken on the spot. Furthermore, the researches which generate and control human's facial images by $\mathrm{CG}$ also performed briskly, and their applications in various fields are expected.

In a human communication scene, it seems that verbal information is the most important and additionally, non-verbal information also plays an important role in natural communication. The facial expression is thought to send the most of the non-verbal information. From this viewpoints many

The original version of this chapter was revised: The copyright line was incorrect. This has been corrected. The Erratum to this chapter is available at DOI: 10.1007/978-0-387-35660-0_65 
researches have been studied such as face-to-face communication using 3D face model, called an avatar on cyberspace as one of the communication forms, and we proposed HYPERMASK as an interaction experiment with theatrical tool using a real object, on which various synthesized facial expressions are projected.

As for speech communication, a spoken language translation has been studied at ATR and a prototype system, ATR-MATRIX[2] is developed for the limited domain of hotel reservation between Japanese and English.

On the other hand, the stand-in of a foreign language for overseas movies and TV programs, has been performed conventionally. But its speech sounds and mouth motions are always not synchronized. Furthermore, the videophone with automatic voice translation are expected to be widely used in the near future, which may face the same problem without lipsynchronization. Lip-synchronized facial image generation is essential for these applications.

This paper proposes a method to generate 3D personal face model with real personal face shape, and to track the face motion like movement and rotation automatically and accurately for audio-visual speech translation and interactive movie system. The method enables to detect translation and rotation of the head by template matching using a 3D personal face model.

In this paper, we describe the method to generate a $3 \mathrm{D}$ personal face model and an automatic face tracking algorithm firstly. And then we introduce a lip synchronization method with synthetic voice and natural voice to realize a speaking image translation system, communication system and an interactive movie system.

\section{3D PERSONAL FACE MODEL}

Face fitting tool developed by our group[3] is a tool to generate a 3D face model using one's photograph. But the manual fitting algorithm requires a lot of time for users to generate a good 3D model with real personal face, although it is able to generate an approximate model every time with real personal shape by fitting many photographs without any lack of facial vertices.

In order to raise accuracy of face tracking using the 3D face model, we used $3 \mathrm{D}$ color range scanner to generate a $3 \mathrm{D}$ model with a real personal shape. We show data acquired by Cyberware range scanner in Fig.1. This data is only a uniform vertices, so we cannot control facial expression because there is no meaning in every point. So at first, to fit a generic face model to the range data, both a generic model and range data are mapped to 2D plane. Then, we manually fit a generic model's face parts to corresponding parts in captured texture (Fig.2 left). Finally, we replace the 
depth coordinates values of the standard model to range data values and obtain a real 3D personal face model (Fig.2 right).
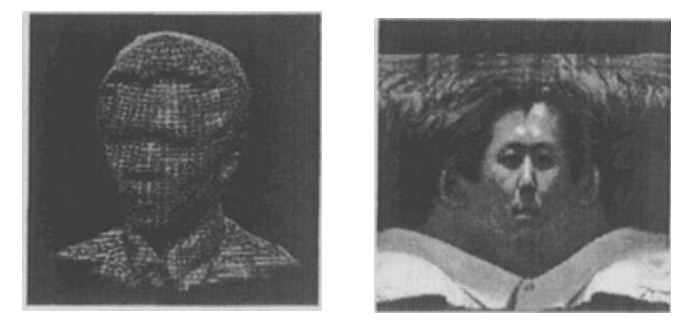

Figure 1. Captured Range Data and Texture
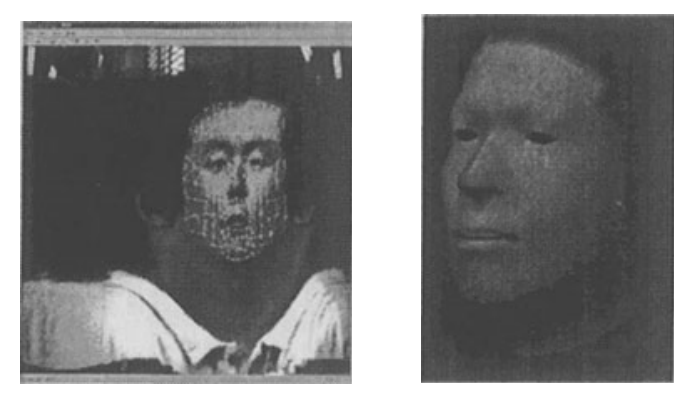

Figure 2. Fitting Result and Generated Personal 3D Model

\section{TRACKING FACE IN VIDEO SEQUENCE}

Many tracking algorithms have been studied by many researchers for a long time, and a lot of algorithms are applied to track a mouth contour, an eyes contour, and so on. However, because of blurring feature points between frames, or occlusion of the feature points by rotation of a head etc., these algorithms were not able to do accurate tracking enough to regenerate natural expression. In this chapter, we describe an automatic face tracking algorithm using a 3D face model. This is not a point tracking but a surface tracking by treating a part of face surface as a rigid object.

Tracking processing using template matching can be divided into the three steps. First, texture mapping of one of the video frame images is carried out to the 3D individual face shape model created in Chapter 2. Here, the frontal face image is chosen out of video frame images for the texture mapping. The reason is that the video images used in this experiment are mainly frontal in movement and the lighting condition does not change so drastically in the 
same video sequence. Anyway, we have to fit a generic face model to one frame shot from video sequence by manual operation.

Next, we make a 2D template images for every translation and rotation using a 3D model (Fig.3). Here, in order to reduce a matching error, a mouth region is excluded in a template image. Thereby, even while the person in a video image is speaking something, tracking can be carried out more stably.

Finally, we carry out template matching between the template images and an input video frame image and estimate translation and rotation values so that a matching error becomes minimum using mean square error of RGB values. Fig.4 shows an example of original video sequence. After face tracking, face part is extracted from original sequence in Fig.5. By replacing with synthesized mouth shape, new image sequence is coming out in Fig.6. In this sequence, he says some sentence other than original.

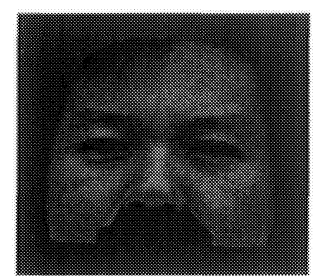

Figure 3. 3D Face Template with Texture from Video

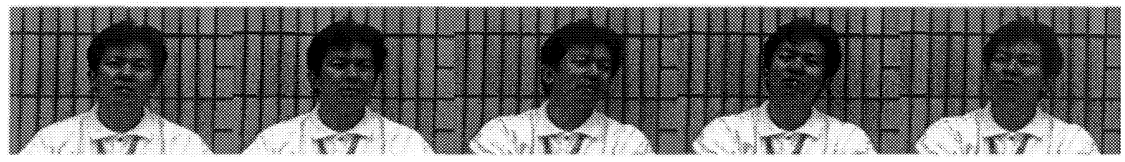

Figure 4. Original Video Sequence

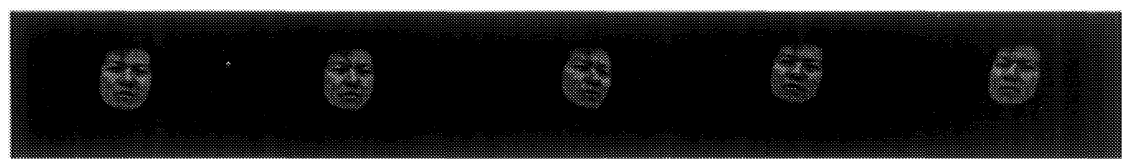

Figure 5. Extracted Face Part after Tracking

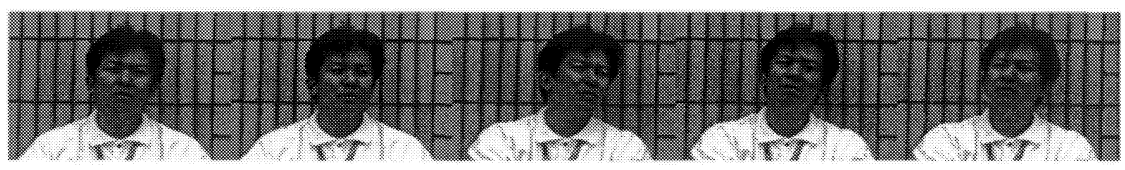

Figure 6. Synthesized Image after Replacing Mouth Part 


\section{VIDEO TRANSLATION SYSTEM}

In this chapter, a video translation system is introduced. The system is divided broadly into two parts: one is a speech-translation part and a imagetranslation part. A speech-translation part is composed of ATR-MATRIX[2], which was developed in ATR-ITL. ATR-MATRIX is composed of ATRSPREC to execute speech recognition, TDMT to handle text-to-text translation, and CHATR to generate synthesized speech. In the process of speech translation, the two parameters of phoneme notation and duration information, which are outputs from CHATR, are applied to facial image translation.

The first step of a image-translation part is to make a 3-D model of the mouth region for each speaker by fitting a standard facial wire-frame model to an input image. Because of the differences in facial bone structures, it is necessary to prepare a personal model for each speaker, but this process is required only once for each speaker. This process is already shown in Chapter 2.

The second step is to generate lip movements for the corresponding utterance. The 3-D model is transformed by controlling the acquired lipshape parameters so that they correspond to the phoneme notations from the database used at the speech synthesis stage. Duration information is also applied and interpolated by some interpolation for smooth lip movement. Here, the lip-shape parameters for each Viseme are defined by a momentum vector derived from a neutral face at lattice points on a wire-frame. Therefore, this database does not need speaker adaptation.

In the final step of a image-translation part, the translated synthetic mouth region's 3-D model is embedded into input images. In this step, the 3-D model's color and scale are adjusted to the input images. Even if an input image sequence is moving during an utterance, we can acquire natural synthetic images because tracking result is accurate and a 3-D face model has personal geometry information. Consequently, the system outputs a lipsynchronized face image to the translated synthetic speech at 30 frames/sec. Fig.7 shows an example of translated image frame shot.
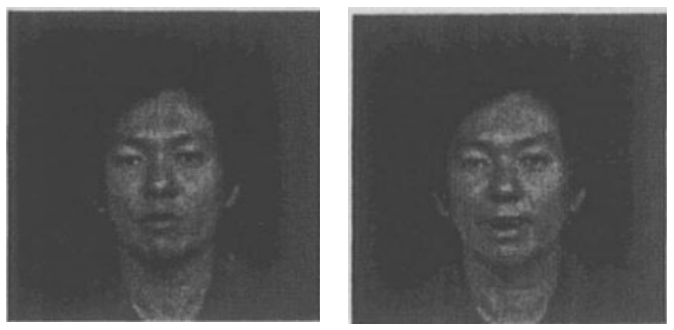

Figure 7. Original and Translated Image 


\section{INTERACTIVE COMMUNICATION SYSTEM}

To realize lip synchronization in an interactive system or communication system, the spoken voice has to be directly analyzed and converted into mouth shape on real-time. So this parameter conversion is realized by a neural network on a frame-by-frame basis.

Prototype of multiple-user communication systems in cyberspace are constructed based on a server-client system. In our system, only a few parameters and the voice signal are transmitted through the network. The avatar's face is synthesized by these parameters locally in the client system.

In the server system, the voice from each client is phonetically analyzed and converted to mouth shape. LPC Cepstrum parameters are converted into mouth shape parameters by a neural network trained by vowel features. Fig.8 shows the neural network structure used for parameter conversion. Twenty dimensional Cepstrum parameters are calculated every $32 \mathrm{~ms}$ with $32 \mathrm{~ms}$ frame length. In the client system, the on-line captured voice of each user is digitized a $16 \mathrm{KHz}$ and 16 bits and transmitted to the server system frame-by-frame through a network in the communication system. Then the mouth shape of each avatar in cyberspace is synthesized by this mouth shape parameter received at each client.

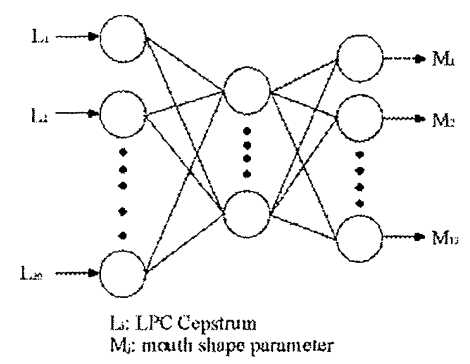

Figure 8. Neural Network for Parameter Conversion

Mouth shape can be easily edited by our mouth shape editor. We can change each mouth parameter to set a specific mouth shape on the preview window. Typical vowel mouth shapes are shown in Figure 9. Our special mouth model has polygons for inside the mouth and the teeth. A tongue model is now considered. For parameter conversion from LPC Cepstrum to mouth shape, only the mouth shapes for five vowels and nasals are defined as the training set. We have defined all of the mouth shapes (Visemes) for Japanese phonemes and English phonemes database by using this mouth shape editor to realize video translation system.

Fig.10 shows a prototype communication system with avatar in cyberspace. Each user can walk through and fly through cyberspace by 

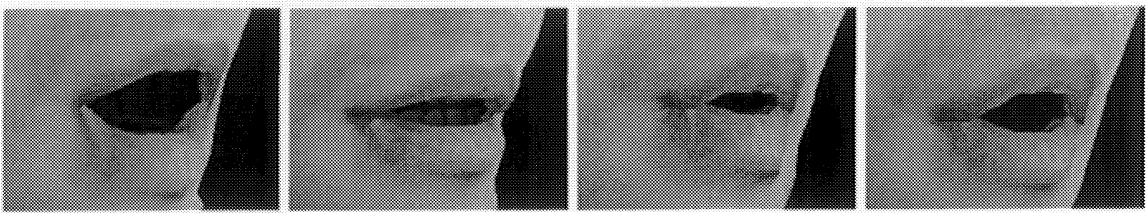

Figure 9. Typical Mouth Shapes for Vowels

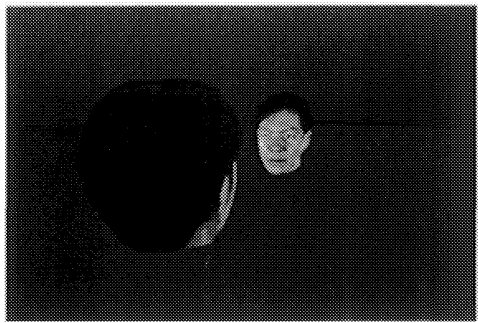

Figure 10. Communication System with Avatar in Cyberspace

mouse control and the current locations of all users are always monitored by the server system. The avatar image is generated in a local client machine by the location information from the server system.

The location information of each avatar, mouth shape parameters, and emotion parameters are transmitted every $1 / 30$ seconds to the client systems. The distance between any two users is calculated by the avatar location information, and the voice from every user except himself or herself is mixed and amplified with gain according to the distance.

Based on facial expression parameters and mouth shape parameters, an avatar's face is synthesized frame-by-frame. Also, the avatar's body is located in cyberspace according to the location information.

When a new user enters the system, his or her face model and voice model have to be registered before operation. To simplify the face model construction and voice learning, a GUI tool for speaker adaptation has been prepared.

\section{INTERACTIVE MOVIE SYSTEM}

When people watch movies, they sometimes overlap their own figure with the actor's image. An interactive movie system we constructed is an image creating system in which the user can control facial expression and lip motion of his or her face image inserted into a movie scene. The user submits a voice sample by microphone and pushes keys that determine 
expression and special effect. His or her own video program can be generated in real-time.

At first, once a frontal face image of a visitor is captured by camera, a 3-D generic wireframe model is fitted onto the user's face image to generate a personal 3-D surface model. A facial expression is synthesized by controlling the grid point of the face model and texture mapping. For speaker adaptation, the visitor has to speak five vowels to choose an optimum weight from the database.

In the interactive process, a famous movie scene is going on and the facial region of an actor or actress is replaced with visitor's face. Facial expression and lip shape are also controlled synchronously by the captured voice. Figure 11 shows the result of face model fitting into original movie scene. Figure 12 shows an user's face inserted into actor's face after color correction. Any expression can be appended and any scenario can be given by the user independent of the original story in this interactive movie system.

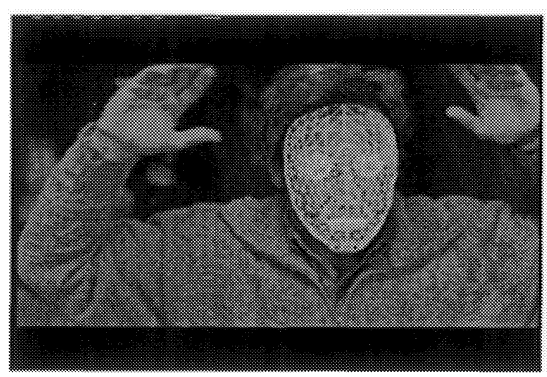

Figure 11. Fitted Model onto Original Scene

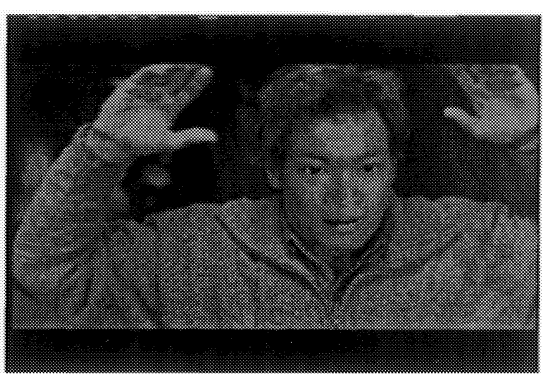

Figure 12. Replaced Face onto Actor's

\section{CONCLUSION}

In this paper, some face image analysis and synthesis method for video translation, communication and interactive movie system. Current method requires many manual operations. So full automatic system is next target of this system. At the same time, robustness in tracking process and image quality of face expression synthesis are also future study.

\section{References}

[1] Shigeo Morishima, "Face Analysis and Synthesis", IEEE Signal Processing Magazine, Vol.18, No.3, pp.26-34, May 2001.

[2] T. Takezawa, et.al., "Japanese-to-English speech translation system:ATR-MATRIX", Proc. of ICSLP, pp. 957-960, 1998.

[3] Facial Image Processing System for Human-like "Kansei" Agent web site : http://www.tokyo.image-lab.or.jp/aa/ipa/ 